Revue internationale P.M.E.

Économie et gestion de la petite et moyenne entreprise

\title{
La complémentarité stratégique entre grande entreprise et PME en matière d'innovation
}

\section{Régis Larue de Tournemine}

Volume 7, numéro 3-4, 1994

URI : https://id.erudit.org/iderudit/1008428ar

DOI : https://doi.org/10.7202/1008428ar

Aller au sommaire du numéro

\section{Éditeur(s)}

Presses de l'Université du Québec

ISSN

0776-5436 (imprimé)

1918-9699 (numérique)

Découvrir la revue

Citer cet article

Larue de Tournemine, R. (1994). La complémentarité stratégique entre grande entreprise et PME en matière d'innovation. Revue internationale P.M.E., 7(3-4), 173-200. https://doi.org/10.7202/1008428ar
Résumé de l'article

Le rôle respectif joué d'une part par les grandes entreprises et, d'autre part, par les PME en matière d'innovation technologique fait l'objet de nombreuses controverses dans la littérature en sciences de gestion. Prolixe sur ce sujet, cette littérature s'est moins intéressée, jusqu'à une date récente, aux interrelations existantes entre firmes de toutes tailles dans le processus d'innovation. Les observations récentes concernant ces interrelations en particulier dans les nouvelles technologies permettent d'avancer qu'il existe en matière d'innovation technologique une complémentarité stratégique entre grandes firmes et PME. Dans une première partie, cet article met en évidence cette complémentarité stratégique entre grandes et petites entreprises dans les industries fondées sur la science (IFS). La troisième partie de cet article est consacrée aux implications de ces analyses en matière de " création " et de " diffusion » de l'innovation dans les IFS 


\title{
La complémentarité stratégique entre grande entreprise et PME en matière d'innovation technologique
}

\author{
Régis LARUE DE TOURNEMINE* \\ IECS Strasbourg
}

\section{MOTS CLÉS}

\author{
Stratégie technologique - Innovation technologique \\ Industries fondées sur la science \\ Complémentarité stratégique entre grandes et petites entreprises \\ Dynamique du processus d'innovation
}

\begin{abstract}
RÉSUMÉ
Le rôle respectif joué d'une part par les grandes entreprises et, d'autre part, par les PME en matière d'innovation technologique fait l'objet de nombreuses controverses dans la littérature en sciences de gestion. Prolixe sur ce sujet, cette littérature s'est moins intéressée, jusqu'à une date récente, aux interrelations existantes entre firmes de toutes tailles dans le processus d'innovation. Les observations récentes concernant ces interrelations en particulier dans les nouvelles technologies permettent d'avancer qu'il existe en matière d'innovation technologique une complémentarité stratégique entre grandes firmes et PME. Dans une première partie, cet article met en évidence cette complémentarité stratégique entre grandes et petites entreprises dans les industries fondées sur la science (IFS). La troisième partie de cet article est consacrée aux implications de ces analyses en matière de "création " et de "diffusion" de l'innovation dans les IFS
\end{abstract}

* Régis Larue de Tournemine est maître de conférences à la Faculté des sciences économiques et de gestion de Strasbourg. Chercheur au Bureau d'économie théorique et appliquée. Directeur du Département logistique-production de l'IECS (Institut d'études commerciales supérieures) Strasbourg. Il est spécialisé dans le domaine des stratégies technologiques et industrielles et dans le management de l'innovation. Adresse : 59, rue du Zürich, 67000 Strasbourg, France. 


\begin{abstract}
In management literature the respective importance of big firms on the one hand and small and medium firms on the other is a highly controversial subject. While management literature has long proved verbose in this area, little attention has been paid, until recently, to the existing relations between firms of all sizes during the innovation process.

Recent studies concerning such relations, particularly in respect of new technologies, lead us to the following conclusion: in the technological process there does indeed exist a strategic complementarity between big and small firms. The first part of this article concentrates on this complementarity. The second part analyses the characteristics and the dynamics of the strategic commplementarity between big and small firms in science-based industries. The third part of the article is devoted to the implications of this analysis concerning technological strategies and the production and distribution of innovations in science-based industries.
\end{abstract}

\title{
RESUMEN
}

El papel respectivo desarrollado por las grandes empresas y por otra parte por las PyME en materia de inovacion technologica es objecto de numerosas controversias en la literatura de ciencias de gestion.

Esta literatura se interesa menos desde hace poco tiempo po las interrelaciones que existen entre las empresas de cualquier dimension en el proceso de inovacion. Las recientes observaciones relativas a estas interrelaciones, en particular en las nuevas tecnologias, permiten decir que en la inovacion existe una complementaridad estratégica entre las grandes y pequenas empresas.

En una primera parte, este articulo pone de relieve esta complementaridad. Una segunda parte define las caracteristicas entre las grandes y pequenas empresas en las industrias fundadas sobre la ciencia. La tercera parte de este articulo se interesa a las implicaciones de estos analisis que se aplican a la "creation" y la "difusion" de la inovacion en las industrias fundadas sobre la ciencia.

\section{Introduction}

Selon plusieurs auteurs (Abernathy et Utterbach, 1978 ; Moore et Tushman, 1982 et Porter, 1982), le processus d'innovation dans un secteur quelconque obéit à une dynamique temporelle selon laquelle l'industrie est conduite d'une structure initiale à une structure potentielle. Il en résulte que la stratégie des firmes est conditionnée par leur clairvoyance concernant l'évolution de leur secteur d'appartenance et les caractéristiques du processus d'innovation de leur industrie. 
Les spécificités de la dynamique du processus d'innovation dans les «nouvelles technologies» (celles qui participent au développement du nouveau paradigme technologique) ont conduit à regrouper les entreprises impliquées dans le développement de ces technologies (biotechnologie, optoélectronique, technologies de l'information, nouveaux matériaux) dans une catégorie à part, les industries fondées sur la science (Pavitt, 1984).

Dans ces industries, l'approche stratégique et le mode de management présentent diverses particularités attestées par de nombreuses analyses. La littérature en gestion avance à ce sujet diverses propositions: modalités particulières du management de l'innovation dans les petites firmes de haute technologie (Horwitch et Pralahad, 1976); management de l'ambivalence entre continuité et changement rapide pour les entreprises de haute technologie (Maidique et Hayes, 1974); rôle central de l'entrepreneur dans les innovations radicales des petites firmes technologiques (Maidique, 1980); etc. Si l'on tire les conclusions de ces diverses propositions, on peut postuler qu'il convient de retenir une approche contingente des stratégies technologiques et du mode de management dans les industries fondées sur la science (Larue de Tournemine, 1991). Dans cette article, un aspect particulier de cette approche contingente sera exploré : celui du management stratégique des complémentarités entre grandes et petites entreprises en matière d'innovation technologique dans les industries fondées sur la science.

La première partie de cet article cherchera à mettre en évidence et à identifier cette complémentarité stratégique entre grandes et petites entreprises. Cette première partie sera fondée sur les recherches en gestion concernant, d'une part, la relation entre taille des entreprises et innovation technologique et, d'autre part, les accords de collaboration industrielle. Une seconde partie permettra de définir les caractéristiques et la dynamique de la complémentarité stratégique entre grandes et petites entreprises dans les industries fondées sur la science. La démonstration de ces caractéristiques et de cette dynamique sera fondée sur des études et les enquêtes effectuées par l'auteur dans le domaine de la photonique et sur des recherches menées aux États-Unis dans le domaine de la biotechnologie. La troisième partie sera consacrée aux implications de ces analyses en matière de stratégies technologiques de création et de diffusion de l'innovation dans les industries fondées sur la science. Cette troisième partie sera illustrée par quelques exemples de collaborations stratégiques entre grandes et petites entreprises. 


\section{Taille des entreprises et innovations technologiques: que conclure des statistiques et des recherches existantes?}

Si l'on considère la multitude des études quantitatives et qualitatives concernant la question des performances comparatives des grandes et des petites firmes en matière d'innovations technologiques, on aboutit vite à la conclusion qu'il n'existe pas de relation simple entre taille des entreprises et performances en matière d'innovations technologiques. Dans une première sous-section (1.1.), les études statistiques existantes et différentes analyses qualitatives concernant le rôle respectif des grandes et petites entreprises en matière d'innovations technologiques nous permettront de conclure que la taille des entreprises n'est pas le seul facteur explicatif. Cette rapide analyse de la littérature et des statistiques existantes nous conduira à identifier d'autres facteurs explicatifs et à avancer qu'il existe une complémentarité entre grande et petite entreprise dans les processus d'innovation technologique. Dans une seconde sous-section (1.2.), cette complémentarité sera explicitée par l'analyse d'un phénomène caractéristique des années 80 : la constitution de réseaux d'entreprises innovantes. L'étude des accords de collaboration entre entreprises dans les années 80 montre que les accords de nature scientifique et technologique sont particulièrement nombreux dans les nouvelles technologies. Dans ces technologies, les avancées scientifiques et techniques reposent en grande partie sur le management des collaborations interfirmes. Cette constatation nous permettra de conclure qu'il est nécessaire de caractériser plus en détail, d'une part, les spécificités du processus d'innovation dans ces nouvelles technologies, d'autre part, les complémentarités stratégiques existantes entre grandes entreprises et PME dans les industries fondées sur la science (IFS).

\subsection{Existe-t-il une relation entre taille des entreprises et innovations technologiques?}

En matière d'innovation technologique, le rôle respectif des grandes firmes et des PME est souvent controversé. Cette controverse peut être illustrée par différentes études.

- Un récent ouvrage consacré à l'économie du changement technique (Le Bas, 1991) conclut que la question des performances comparatives des grandes et petites firmes en matière d'innovation n'a ni «solution évidente» sur la base de la réflexion théorique, ni «début de vérification empirique » à partir des statistiques existantes 
concernant les dépenses de R-D et le nombre de brevets déposés. Cet ouvrage qui reprend les travaux de multiples auteurs aboutit à un constat nuancé concernant, d'une part, la relation entre intensité de la recherche et taille des firmes et, d'autre part, la relation entre efficacité des recherches et taille des firmes.

1. En ce qui concerne l'existence d'une relation positive entre intensité de la recherche et taille des firmes (thèse de Schumpeter et Galbraith), la corrélation semble faible, et il convient de tenir compte des spécificités économiques et technologiques sectorielles. Cette conclusion s'impose même aux auteurs d'un travail de synthèse à partir de nombreuses études empiriques (Kamien et Schwarz, 1975) qui avancent que l'effort de recherche s'accroît avec la taille des firmes.

2. Quant à la relation entre efficacité des recherches et taille des firmes, la réponse est également nuancée. L'existence de rendements d'échelle croissants de la recherche défendue par Schumpeter et Galbraith a été remise en perspective par diverses recherches empiriques. Certains auteurs (Kamien et Schwarz, 1975) concluent ainsi à une grande efficacité innovatrice des PME par unité monétaire dépensée en R-D, tandis que l'on peut démontrer (Zuscovitch, 1984) qu'il n'existe pas une loi générale de rendements croissants des dépenses de R-D mais plutôt des «segments » de rendements croissants liés au phénomène «d'indivisibilité » dans des secteurs particuliers (chimie et pharmacie, par exemple). Il est évident que cette dernière proposition revient à réintroduire les spécificités économiques et technologiques sectorielles comme paramètres explicatifs supplémentaires dans la relation entre efficacité des recherches et taille des firmes.

- Une analyse (Rothwell, 1985) à partir de la banque de données du SPRU (Science Policy Research Unit) concernant 4400 innovations de produits dans l'industrie britannique de 1945 à 1983 aboutit également à un bilan nuancé.

1. Premièrement, le rôle respectif joué par les grandes entreprises et les PME en matière d'innovation évolue historiquement. Ainsi, le pourcentage du nombre total d'innovations réalisées par des firmes de moins de 500 salariés a augmenté de $26,4 \%$ en 38 ans. Sur cette période, on constate de plus qu'une proportion croissante d'innovations est le fait de filiales de grandes firmes diversifiant leurs activités par croissance externe. 
2. Deuxièmement, le rôle respectif joué par les grandes entreprises et les PME en matière d'innovation dépend fortement du secteur considéré et varie souvent chronologiquement. Le taux d'innovation des PME sur la période étudiée est relativement stable dans l'instrumentation scientifique (58\%): dans la pharmacie; il passe de $14 \%$ avant 1974 à $0 \%$ de 1974 à 1983 ; il augmente fortement dans l'informatique et les technologies de l'information (faible avant $1970 ; 3 \%$ de 1970 à $1974 ; 47 \%$ de 1975 à $1979 ; 64 \%$ de 1980 à 1983). Ces variations du taux d'innovation des PME reflètent probablement des changements institutionnels (durée et coût de l'homologation des médicaments dans la pharmacie) et des évolutions en matière de technologies et d'indivisibilité dans l'informatique et les technologies de l'information (fabrication de grands ordinateurs centraux avant 1970, mini-informatique à partir de 1970, micro-informatique à partir de 1980).

3. Troisièmement, les grandes entreprises et les PME ont des rôles différenciés dans le processus d'innovation spécifique à chaque secteur. En particulier, des analyses plus qualitatives de Rothwell concernant la dynamique du processus d'innovation dans différentes activités (semi-conducteurs et CAO plus spécialement) montrent que les grandes firmes sont «technologiquement plus inventives» (elles développent le nouveau paradigme technologique) tandis que les petites firmes diffusent le nouveau paradigme en concevant de nouvelles applications et en créant de nouveaux marchés.

En définitive, Rothwell postule également l'existence de spécificités sectorielles et ajoute des considérations d'évolution économique, de dynamique technologique et de complémentarité entre petites et grandes entreprises pour expliciter l'impact de la taille des firmes sur leur efficacité en termes d'innovation.

- Divers tests effectués à partir de données d'entreprises françaises (Crepon, 1991) ont également cherché à vérifier pour la France la conjecture de Schumpeter, attribuant un plus grand dynamisme technologique aux grandes entreprises monopolistiques. Ces tests montrent que la décision de faire de la recherche implique une taille minimale. Mais à partir de cette taille, la relation entre efforts de recherches et taille prend la forme d'une courbe en $U$.

- D'autres auteurs (Acs et Audretsch, 1988) posent la question sous une forme différente en se demandant quelles sont les conditions qui favorisent l'activité innovatrice des grandes firmes, d'une part, et des 
petites firmes, de l'autre. Ces deux auteurs montrent que les grandes firmes innovent le plus dans les industries concentrées et fortement capitalistiques, tandis que les petites firmes sont plus innovatrices dans les industries jeunes caractérisées par une forte dispersion des tailles relatives des entreprises.

Si l'on veut résumer l'ensemble de ces recherches, on peut conclure qu'en matière d'innovation, les rôles respectifs joués par les grandes entreprises et par les PME résultent d'un jeu d'influences complexe qui comporte divers aspects :

- Les spécificités sectorielles à un moment donné; en particulier, les phénomènes «d'indivisibilité technologique » en matière de R-D jouent, à l'évidence, un rôle déterminant dans la position respective des grandes et des petites firmes dans le processus d'innovation à une date donnée (point de vue statique).

- L'évolution de ces spécificités sectorielles (point de vue dynamique). On constate, par exemple, que dans le domaine des technologies de l'information, la contribution des petites entreprises à l'innovation technologique a considérablement augmenté dans les années 60,70 et 80 . Nous avons attribué cette évolution à la réduction des «indivisibilités technologiques » induites par le passage de la production de grands ordinateurs centralisés, aux mini-ordinateurs puis à la micro-informatique.

- La dotation en «avantages comparatifs» du point de vue de la capacité innovatrice selon la taille à un moment donné (point de vue statique); avantages en ressources financières et techniques pour les grandes entreprises; avantages de souplesse, d'adaptabilité et de rapidité de réponse pour les petites firmes.

- L'évolution de cette dotation en «avantages comparatifs» (point de vue dynamique). Par exemple, sur la période 1970-1990, les grandes firmes gagnent en flexibilité organisationnelle (intraprenariat, création de petites unités autonomes au sein des grandes structures industrielles, nouvelle organisation du développement de produits nouveaux et de la production en général, etc.), tandis que les petites firmes accèdent plus facilement aux ressources financières et technologiques du fait de l'évolution des «Systèmes Nationaux d'Innovation» (parcs scientifiques et technologiques, capital risque, second marché, etc.).

- Le fait que les grandes entreprises et les PME ont des rôles différenciés dans le processus d'innovation spécifique à chaque secteur. On peut ainsi postuler l'existence d'une certaine complémentarité entre 
grandes et petites entreprises (Rothwell, 1985). Rothwell affirme ainsi que « le point final, et sans doute le plus important, est que les petites et les grandes entreprises ne vivent pas dans un monde séparé. Au contraire, dans une grande variété de secteurs, s'instaurent des flux complexes de savoir-faire technologique et d'innovation entre les firmes de toutes tailles ». Ce point de vue rejoint, par ailleurs, de nombreuses analyses récentes du processus d'innovation conçu comme le résultat d'un acte collectif auquel participe une foule d'intervenants diversifiés (Callon et Latour, 1985; Akrich, Callon et Latour, 1988). Dans cette optique, l'essor de «réseaux d'entreprises innovantes » dans les années 80 peut être analysé afin de mieux spécifier la mise en œuvre industrielle de ces complémentarités entre grandes et petites entreprises sous la forme de la multiplication des accords de collaboration entre entreprises.

\subsection{Accords de collaboration industrielle et complémentarité entre grandes et petites entreprises}

Dans les années 80, les collaborations entre entreprises ont connu un essor considérable et les recherches théoriques et empiriques concernant ces collaborations constituent maintenant un domaine très actif des sciences de gestion. Les pages qui suivent se limiteront à retenir quelques conclusions de ces recherches dans le domaine des nouvelles technologies et des relations particulières qui se sont établies entre grandes entreprises et PME dans ce domaine. L'essentiel des données quantitatives utilisées sont celles de la banque de données CATI (Cooperative Agreements and Technology Indicators) établi par le MERIT (Université de Maastricht) qui concerne exclusivement des accords de nature scientifique ou technologique (10 000 accords concernant 3500 entreprises).

Dans l'optique de la complémentarité stratégique entre grandes et petites entreprises en matière d'innovations dans les technologies nouvelles, nous nous limiterons à retenir les conclusions qui suivent.

1. L'essor des nouvelles technologies a considérablement stimulé les collaborations: par exemple, dans l'industrie pharmaceutique, les conditions concurrentielles ont été profondément modifiées par l'apparition des biotechnologies (NGS, Pearson et Ball, 1992). Dans l'industrie pharmaceutique, les biotechnologies ont été à l'origine de l'entrée de nouvelles petites entreprises, tandis que proliféraient les collaborations en matière de $\mathrm{R}-\mathrm{D}$ entre ces petites entreprises nouvellement constituées (firmes émergentes) et les grands groupes pharmaceutiques (firmes établies). 
Au cours des années 80 , la banque de données CATI montre que les technologies de l'information totalisent la plus grande part des alliances technologiques $(41 \%)$; suivent les biotechnologies $(20 \%)$ et les nouveaux matériaux (10\%) (Hagedoorn, 1993). Pour Hagedoorn, ce sont les technologies du nouveau «paradigme technologique » qui expliquent l'essor des alliances technologiques dans les années 80 . Parmi ces trois technologies nouvelles (information, biotechnologie, nouveaux matériaux), c'est dans le domaine des technologies de l'information que le réseau d'alliances est le plus dense.

2. Les collaborations en matière de $R-D$ sont souvent limitées à des domaines "précompétitifs»(Dodgson, 1992). La croissance des recherches conjointes a été particulièrement significative à partir de 1975 (Hladik, 1985).

3. Les capacités managériales d'identification des meilleurs partenaires possibles et de négociation avec ces partenaires sont devenues un élément essentiel pour la réussite des stratégies technologiques des entreprises. En effet, dans les technologies de l'information, les avantages concurrentiels obtenus par de nombreuses firmes résultent souvent de leur capacité à identifier les «bons » partenaires puis à négocier avec eux (Littler et Wilson, 1990). Le management de ces collaborations est très difficile et il a un impact déterminant sur les résultats scientifiques, technologiques et industriels obtenus (Dodgson, 1992).

4. Ces collaborations constituent, pour les grandes firmes établies, un moyen efficace de restructurer leurs activités et d'améliorer leur vision stratégique. Dans les années 80 , les collaborations entre firmes sont des vecteurs de réorientations stratégiques tandis que se multiplient les processus d'innovation interfirmes (par opposition aux développements innovatifs purement internalisés). Ces collaborations permettent aux grandes firmes de s'assurer une position d'observateur privilégié des nouveaux développements technologiques en limitant les investissements dans les recherches dont les résultats sont encore très «spéculatifs». Cette stratégie d'observation (wait and see strategy) a été, en particulier, celle des grands groupes pharmaceutiques à l'égard des petites firmes biotechnologiques (Dodgson, 1992). Ces alliances stratégiques sont des moyens efficaces de restructuration interne lorsque technologies et marchés évoluent rapidement et mettent en péril les positions concurrentielles existantes. Dans ce cas, les alliances stratégiques constituent une alternative à la cessation pure et simple d'activités pour des firmes établies sur des technologies devenues obsolètes (Benassi, 
1993). Les grandes entreprises se sont ainsi intéressées à des formes nouvelles de partenariat avec des PME, afin d'exercer une veille technologique. Cette politique de partenariat établie par les grandes entreprises a été, en particulier, mise en œuvre dans le cadre de l'essor du capital de risque: constitution par les grandes firmes de filiales financières spécialisées dans le capital de risque, prise de participation de grands groupes industriels dans des PME lorsque la société de capital-risque est en phase de désengagement (Geoffron, 1991). En fait, les modalités de ces stratégies d'alliances technologiques sont multiples (recherches conjointes, prise de participation, contrats de R-D, accords d'échanges technologiques, licences, etc.) et définissent différents degrés d'interdépendance organisationnelle (Hagedoorn, 1993).

En définitive, les études concernant les accords de collaboration entre firmes montrent bien que l'essor des nouvelles technologies impliquent, aussi bien pour les grandes firmes établies que pour les petites firmes émergentes, une multitude de relations d'échanges et de travail en commun ad hoc souvent dans des domaines précompétitifs. L'exemple de ces relations est bien illustré par le cas des programmes européens de développement des «technologies génériques » (Eureka, Esprit, Eurolaser, etc.) dans les années 80. Dans le cadre de ces programmes de développement des «technologies génériques», les succès escomptés reposent en partie sur les capacités de management en matière de R-D partiellement externalisées. Pour les grandes firmes établies, ces capacités de management ont conditionné leur aptitude à restructurer leurs activités, tandis que pour les petites firmes émergentes, les enjeux stratégiques consistent à triompher de leurs limites en matière de ressources disponibles: ressources financières, technologies complémentaires, savoirfaire en matière de systèmes. Du point de vue des stratégies technologiques, mises en œuvre par les grandes firmes établies et par les petites firmes émergentes dans les nouvelles technologies, les accords entre entreprises permettent de tirer parti des complémentarités stratégiques existantes dans les industries fondées sur la science (Larue de Tournemine, 1991). Les spécificités et la dynamique du processus d'innovation dans ces industries fondées sur la science doivent être explicitées, plus en détail, si l'on veut comprendre les fondements théoriques (évolution de la situation de concurrencecoopération) et concrets (stade d'élaboration des connaissances scientifiques et des réalisations technologiques) qui permettent de définir divers stades de complémentarités stratégiques entre grandes firmes établies et petites firmes émergentes. 


\section{Spécificités du processus d'innovation dans les industries fondées sur la science (IFS) et complémentarité stratégique entre grandes entreprises et PME}

Les industries fondées sur la science (optoélectrotechnique, biotechnologie, nouveaux matériaux, technologies de l'information) ont pour particularité de se constituer progressivement à partir d'avancées scientifiques et technologiques majeures qui fondent le nouveau système technique (au sens de B. Gille) en émergence. Dans cette seconde partie, nous préciserons le concept d'industries fondées sur la science avant d'analyser le processus d'innovation spécifique à ces industries. Cette analyse de la dynamique du processus d'innovation dans les IFS nous permettra de caractériser la complémentarité existante entre grandes entreprises et PME.

\subsection{Le concept d'IFS}

Le concept d'industrie fondée sur la science est issu d'un article (Pavitt, 1984) qui analyse les modalités sectorielles du changement technique et des processus d'innovation. Le cadre théorique de cette analyse est celui de la théorie évolutionniste du changement technique. En effet, l'auteur identifie des «trajectoires technologiques» similaires selon une typologie sectorielle établie à partir d'une base de données statistiques portant sur 2000 innovations significatives recensées entre 1945 et 1979 par le SPRU (Science Policy Research Unit, Université de Sussex) en Grande-Bretagne. Cette typologie regroupe les différents secteurs en quatre catégories caractérisées chacune par des trajectoires technologiques identiques.

La trajectoire technologique des industries fondées sur la science (les industries émergentes du nouveau système technique) comporte trois caractéristiques :

- origine ou source de la technologie: R-D et connexion avec les institutions de recherches fondamentales (universités et laboratoires publics);

- besoin des utilisateurs et caractéristiques de la demande: la grande diversité des innovations issues des IFS satisfait une multitude de besoins, dans l'ensemble des secteurs d'activité du système économique (les technologies nouvelles sont «transversales", c'est-à-dire qu'elles trouvent des applications dans de très nombreux secteurs différents). Les IFS répondent à une demande de performances fonctionnelles plus élevées (par rapport à celle des produits existants sur 
la base des technologies traditionnelles), caractéristiques du changement de paradigme technologique;

- appropriation des bénéfices de l'innovation: selon des modalités mixtes qui combinent brevets, secrets, avances technologiques et compétences spécifiques.

Ces analyses en termes de trajectoires technologiques permettent de spécifier des comportements innovatifs et des approches de management contingentes, c'est-à-dire spécifiques à des secteurs déterminés. Certains auteurs (Ducos et Joly, 1988) ont, par exemple, adopté cette approche en termes de trajectoire technologique pour analyser le rôle des biotechnologies dans la mutation actuelle du système industriel. Ces analyses permettent de définir les caractéristiques (managériales, institutionnelles, etc.) de l'émergence d'une nouvelle trajectoire technologique (les biotechnologies dans le cas cité) et l'incidence des biotechnologies sur les caractéristiques des trajectoires existantes dans divers secteurs plus traditionnels (chimie, pharmacie, agrochimie, industrie des semences). Parmi ces incidences, retenons en particulier les coopérations interfirmes dans le cadre de la création de nouvelles technologies et le jeu de concurrence/coopération entre les NEB (nouvelles entreprises de biotechnologie) et les entreprises déjà établies. Le concept d'IFS a été également utilisé afin de préciser les spécificités et la dynamique du processus d'innovation dans les nouvelles technologies: optoélectronique ou photonique (Larue de Tournemine, 1991), biotechnologie (Hamilton, 1985 et 1988).

\subsection{Spécificités et dynamique du processus d'innovation dans les IFS: l'exemple de la photonique et des biotechnologies}

On regroupe sous le terme de photonique (ou optoélectronique), un ensemble de technologies transversales regroupant les lasers, les fibres optiques et les systèmes de visualisation. Cet ensemble de technologies trouve sa cohérence dans l'étude scientifique des phénomènes d'interaction lumière-matériau (la photonique au sens de sciences de photon). La photonique est à la fois complémentaire du développement de la micro-électronique (elle permet d'élargir et d'enrichir les possibilités d'utilisation de la micro-électronique) et concurrente de la micro-électronique (elle a un potentiel de performances plus élevé). De plus, la photonique permet d'envisager la transformation de la matière (utilisation des lasers de forte puissance pour l'usinage). Les enjeux industriels de la photonique sont considérables aussi bien à court terme qu'à long terme (Brendlé, Cohendet, Gaffard et Larue de Tournemine, 1983).

Pour illustrer les spécificités et la dynamique du processus d'innovation dans les IFS, nous reprendrons, ci-dessous, quelques conclusions de recher- 
ches concernant les stratégies industrielles dans les technologies laser. Ces recherches effectuées dans les années 80 (Larue de Tournemine, 1984 ; 1988) ont impliqué toute une série d'enquêtes dans les milieux scientifiques (laboratoires publics et privés, experts) et industriels (petites firmes à base scientifique et technologique, groupes industriels diversifiés).

Dans le cas des technologies laser, ces études longitudinales concernant les évolutions scientifiques, technologiques et industrielles montrent l'existence de différentes phases du point de vue des caractéristiques du processus de l'innovation:

\section{Les années 60}

À la suite des travaux de Gould, dont la thèse (1957) à l'Université de Columbia porte sur les calculs de faisabilité d'un laser, de multiples programmes de développement débouchent sur divers prototypes de laser (Gaillard, 1990) : 1960 (laser à rubis au laboratoire de Hughes Research) ; 1960 (laser hélium néon au laboratoire Bell) ; 1961 (laser au verre néodyme) ; 1964 (laser argon et laser $\mathrm{CO}_{2}$ ) ; 1966 (laser à colorant). En d'autres termes, les années 60 sont caractérisées par de nombreuses recherches sur la faisabilité de l'effet laser dans différents milieux ambiants qui donneront lieu à une multitude de lasers, classés selon la nature de ce milieu (laser à gaz, à colorant liquide, à solide). Un grand nombre de recherches sont alors orientées vers des avancées scientifiques : études de plasma, principe de fusion inertielle, compréhension des phénomènes d'interaction lumière-matériaux. En somme, les efforts dans les années 60 sont orientés vers la réalisation des avancées scientifiques nécessaires à la définition d'un champ de recherches susceptible de contribuer par la suite au développement des technologies.

\section{Les années 70}

Ces années sont consacrées à de multiples développements technologiques concernant les performances des sources lasers existantes (durée de vie, fiabilité, stabilité), la réalisation de nouvelles sources et le développement des technologies associées (optique, informatique, micro-informatique, etc.). Au cours des années 70, le marché des lasers a connu un premier essor sur le plan mondial, mais essentiellement soutenu par des commandes publiques massives souvent militaires. Pour les marchés concurrentiels civils en France, les efforts de la CILAS (groupe CGE), en matière de laser $\mathrm{CO}_{2}$ conduisent en 1978 au développement, avec la SAF (soudure autogène française), d'un système de découpe d'une puissance de $300 \mathrm{~W}$ qui sera vendu à trois exemplaires et dont la conception sera entravée, en particulier, par l'inadaptation des technologies associées (performances insuffisantes des commandes 
informatisées). La combinaison de l'optique et de l'électronique constitue une difficulté récurrente dans les développements des systèmes optoélectroniques: "Même aux États-Unis, dans les années 70, le développement complet d'un projet de machine laser pouvait prendre près de dix ans, comme le démontre, par exemple, la découpe/soudure des boîtes de poisson chez American Can.» (Gaillard, 1990) En d'autres termes, dans les années 70, la concurrence porte sur le développement des technologies nécessaires à la réalisation de systèmes laser: technologie des sources et technologies associées. $\mathrm{Si}$, au cours de cette période, diverses expériences industrielles concernent bien les applications en usinage laser, la technologie laser (technologie des sources et technologies associées) n'acquerra que progressivement les caractéristiques industrielles nécessaires pour la réalisation de systèmes d'usinage $\mathrm{CO}_{2}$ et YAG adaptés aux besoins des utilisateurs : fiabilité de la source et des optiques, qualité du faisceau, adaptation des technologies associées et de l'informatique à l'usinage laser.

\section{Les années 80}

Le marché civil du laser d'usinage commence à se structurer et les industriels offrent non seulement des sources industrielles fiables $\left(\mathrm{CO}_{2}\right.$ et YAG), mais des solutions aux besoins des industriels sous forme de systèmes, puis de centres d'usinage laser réellement adaptés aux applications. Le marché connaît un essor substantiel sur la base d'une réelle maîtrise des technologies des sources et des technologies associées et d'une extension des applications (découpe tout d'abord, puis soudage, et, à plus long terme, traitement de surface). Le marché global des lasers se caractérise par la diversité des applications, des sources et des systèmes (Larue de Tournemine, 1988). Des «conceptions dominantes » de sources laser s'imposent, selon les applications considérées (Larue de Tournemine, 1988). Par exemple, pour l'usinage des matériaux métalliques, les sources $\mathrm{CO}_{2}$ à flux axial rapide ouvrent la voie, dans la première moitié des années 80 , de la compacité et des puissances supérieures à $500 \mathrm{~W}$ (jusqu'à $10 \mathrm{~kW}$ ) pour de multiples applications (découpe, soudage, traitement de surface). Les paramètres de la concurrence se déplacent de la technologie vers la production (conception modulaire des systèmes laser) et la commercialisation (adaptation des systèmes d'usinage laser à la diversité des besoins du marché, intégration laser-robotique).

En guise de conclusion, on peut retenir que la détermination de ces trois phases dans le processus d'innovation des IFS permet d'expliciter comment un ensemble d'avancées scientifiques et technologiques s'organisent progressivement pour donner naissance à toute une série d'innovations de produits 
(les sources laser et les systèmes laser qui combinent sources et technologies associés dans des produits complexes) et de processus (les technologies de production de ces sources et de ces systèmes laser). Ces innovations de produits et de processus sont à l'origine d'un ensemble d'activités «réellement industrielles », c'est-à-dire régulées par les mécanismes du marché au début de la troisième phase. En effet, au début des années 80, l'offre (la structure industrielle, les produits, les procédés) et la demande (les marchés, les applications) commencent à être clairement identifiées.

- L'offre de laser est répartie entre trois types de participants. D'abord, on retrouve des entreprises de taille moyenne spécialisées dans une grande diversité de sources et de systèmes laser (Coherent et Spectra Physics, en particulier) qui ont internationalisé leurs activités et gèrent de nombreux accords avec d'autres entreprises. Ces entreprises sont nées pour la plupart aux États-Unis au cours de la phase 1 et 2 du processus d'innovation. Viennent ensuite des petites firmes spécialisées dans un petit nombre de sources ou de systèmes laser. Ces petites firmes sont d'origine plus récente et se sont positionnées sur la base d'une avancée technologique particulière (Électrox, par exemple, qui a développé en Grande-Bretagne le premier laser à flux axial rapide et a licencié son système à une grande firme américaine pour le commercialiser aux États-Unis). Enfin, de grands groupes diversifiés ont une activité laser encore marginale; mais par le biais d'une petite activité interne et de divers accords, elles sont susceptibles de prendre position sur le marché.

- La demande de laser commence à se structurer sous l'influence des mécanismes du marché. En effet, si en 1981, le marché mondial des lasers (1 268 millions de dollars) est encore fortement public (70\% du marché dont $50 \%$ pour les dépenses militaires), les marchés civil et commercial deviennent significatifs (30\%). Sur le marché civil, les quatre principales applications (usinage laser, applications scientifiques, mémoires optiques et laser médical) relèvent de technologies et de métiers industriels clairement identifiés. Si l'on prend le cas du marché civil du laser d'usinage, les technologies des sources sont bien définies $\left(\mathrm{CO}_{2}\right.$ et YAG); les sources sont fiables (par rapport aux années 60 et 70 ). Les technologies associées (électronique de commande, système optique de transfert du rayon, etc.) ont été développées spécifiquement pour ce marché. La combinaison des technologies des sources et des technologies associées définit toute une gamme de systèmes laser, puis de centres d'usinage laser réellement adaptés (type, fiabilité et vitesse d'usinage) aux besoins de la demande et aux divers types d'applications (marquage, perçage, découpe, soudure). 
La concurrence par les prix devient réelle et la conception des produits et des processus commence à être réellement industrielle (la conception modulaire des sources date de la première moitié des années 80 ).

Dans les biotechnologies, un schéma d'évolution analogue pour le développement des technologies et des marchés peut être également identifié (Hamilton, 1985). Dans le cas des biotechnologies, le début de ce schéma est plus tardif. Le décalage avec la photonique est d'environ dix ans.

Selon Hamilton (1988), le processus d'innovation, dans une entreprise émergente, est articulé en trois phases distinctes.

\section{Phase 1}

Le processus d'innovation est «scientifique» (la priorité est à la recherche scientifique). Cette phase suit l'avance technologique majeure initiale (la démonstration de la faisabilité de divers lasers à la suite des travaux de Gould, au début des années 60 , pour l'optoélectronique; la recombinaison des molécules de DNA au début des années 70 dans les biotechnologies). L'avance technologique initiale suscite de nombreuses recherches scientifiques. Chaque avancée scientifique soulève de nouvelles questions et ouvre des voies prometteuses qui attirent les financements pour préciser les «options possibles». Ce schéma est bien illustré par les mécanismes du capital-risque dans le cas de Genentech et de nombreuses autres firmes émergentes dans le cas des biotechnologies aux États-Unis (Ducos et Joly, 1988). La concurrence entre firmes repose sur les avancées scientifiques.

\section{Phase 2}

Le processus d'innovation est «technologique» (la priorité est au développement technologique et à la standardisation). Les avancées scientifiques cèdent le pas à de multiples développements technologiques, comme nous l'avons vu pour le laser dans les années 70 et comme c'est le cas selon Hamilton pour les biotechnologies dans les années 80. Les prototypes et les expériences en laboratoire donnent lieu à la conception de systèmes nouveaux et aux développements des technologies. Les efforts des entreprises se portent vers la réalisation des premiers produits et des techniques préindustrielles de production. Cette phase concerne toutes les réalisations nécessaires entre la transition de la connaissance scientifique et les premières réalisations technologiques susceptibles de répondre à des applications spécifiques à titre de démonstration expérimentale, dans un premier temps. Dans le cas des systèmes laser d'usinage, il s'agit des premières machines testées dans les laboratoires de recherche et les bureaux, des méthodes de l'industrie automobile, par exemple, dans les années 70 . 


\section{Phase 3}

Le processus d'innovation est «commercial» (la priorité est au développement commercial, aux interactions avec les utilisateurs, à l'extension des applications). Du côté de l'offre, cette phase est caractérisée par une augmentation des activités innovatrices avales (activités d'industrialisation, de commercialisation et de distribution). Les premiers produits et systèmes commercialisés sont à l'origine de nombreuses «informations » sur l'orientation à donner aux recherches et aux développements technologiques ultérieurs. Pour certains produits ou systèmes, dont la «conception dominante» est maintenant assurée; la production en petites séries et la conception modulaire peuvent être envisagées, comme c'est le cas pour les machines d'usinage laser à source $\mathrm{CO}_{2}$ dans la gamme des puissances inférieures à $5 \mathrm{~kW}$, à la fin des années 80 , et pour les machines YAG de puissance inférieure à $1 \mathrm{~kW}$, à la même époque.

Ce schéma du processus d'innovation en trois phases est représenté par Hamilton (1988) dans le graphique 1.

\section{GraphiQue 1}

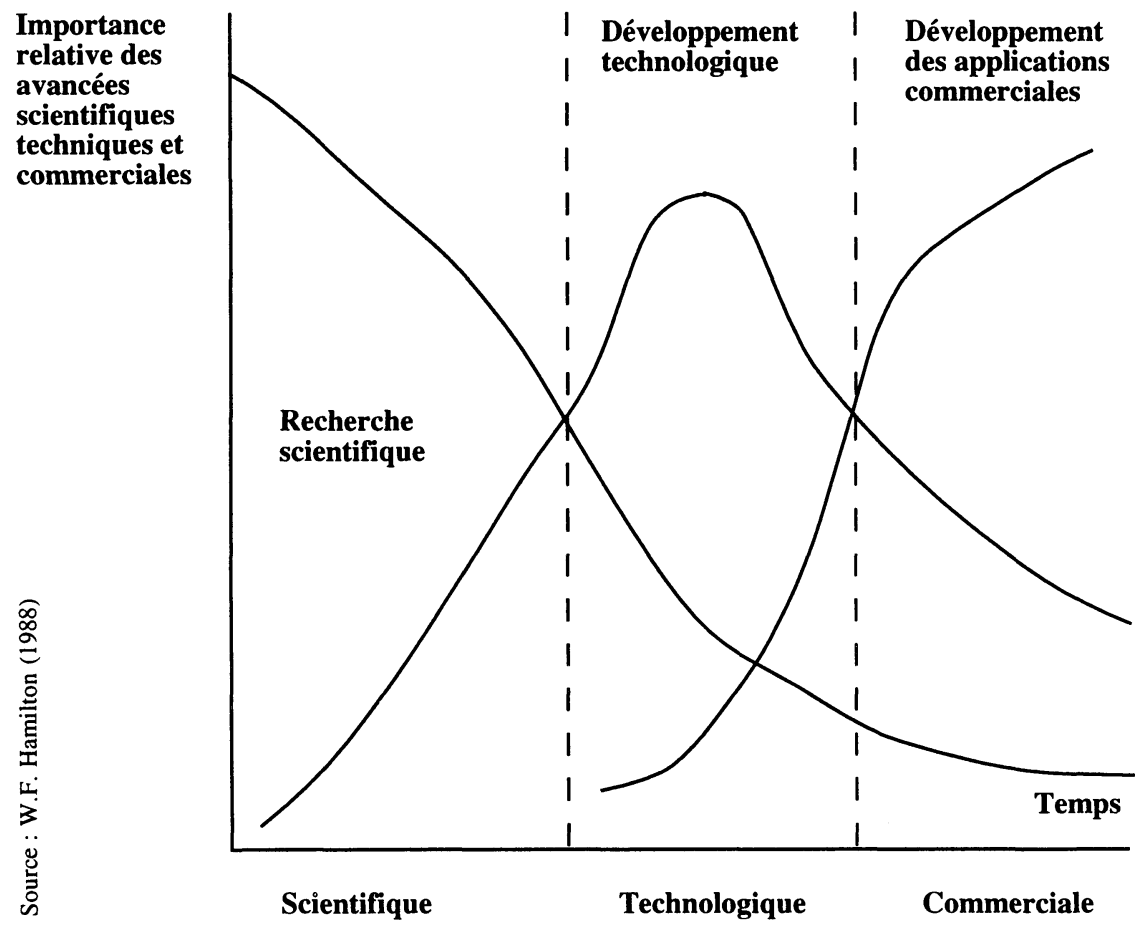


En conclusion, les discontinuités technologiques et la dynamique du processus d'innovation dans les IFS ont de multiples implications stratégiques. Pour les entreprises déjà établies, les discontinuités technologiques sont à l'origine «de menaces » et «d'opportunités » qui sont le résultat d'un long processus scientifique, technologique et commercial : l'identification de ces «menaces» et «opportunités » peut être effectuée progressivement dans la mesure où la discontinuité technologique se traduit en des menaces et opportunités commerciales réelles à l'issue d'une longue période de temps nécessaire à la définition des technologies, et à leur intégration en produits et systèmes susceptibles de répondre à des besoins spécifiques du marché. Pour identifier la «réalité » et la «nature» de ces «menaces et opportunités», la conclusion d'accords avec des petites entreprises émergentes est une manœuvre appropriée.

Pour les entreprises émergentes qui participent dès la première phase (scientifique) à la définition des savoirs scientifiques puis des technologies, des marchés et des règles du jeu concurrentiel, la conclusion d'accords avec les grandes entreprises établies permet d'assurer progressivement leur «structuration » en tant qu'organisation industrielle.

Cette structuration des petites firmes émergentes implique le développement des diverses fonctions (R-D, GRH, finance, production, marketing) qui leur permettront de se constituer en une entreprise autonome en concordance de phase avec la dynamique d'évolution des technologies et des marchés (Larue de Tournemine, 1991). Ce processus de «structuration» met en œuvre des mécanismes de sélection selon lesquels certaines entreprises disparaîtront. D'autres seront absorbées ou contrôlées par les grandes entreprises tandis qu'une minorité assureront leur croissance de façon plus autonome. Ce processus de structuration repose, en fait, sur une évolution des caractéristiques de la situation de complémentarité stratégique entre grandes entreprises et PME. Cette évolution est caractérisée par une modification du rapport entre «opportunités de coopération » et «situation de concurrence» lorsqu' on se déplace de la phase 1 (scientifique) à la phase 3 (commerciale) d'évolution des technologies et des marchés. En effet, les motivations qui ont conduit à la signature de ces accords vont perdre de leur intensité avec le temps.

- Les incertitudes concernant les technologies, les produits, les marchés et les besoins des utilisateurs diminuent.

- Les grandes entreprises établies ont accumulé les savoirs et les apprentissages dont elles avaient besoin. 
- La taille des petites entreprises a augmenté et elles disposent de ressources plus importantes. Elles n'ont plus le même besoin d'accéder aux actifs complémentaires (Teece, 1986) que leur offraient les grandes firmes. En effet, elles disposent de ces actifs complémentaires (réseau commercial, technologies associées, etc.) soit de façon interne, soit par des accords déjà établis.

- Un certain nombre de petites entreprises ont disparu ou ont été absorbées.

Tandis que les incitations à établir des coopérations diminuent, la situation devient de plus en plus concurrentielle sur les marchés et les applications qui sont identifiés dans la phase 3 (commerciale). La complémentarité stratégique entre grandes et petites entreprises diminue ainsi progressivement lorsqu'on passe de la phase 1 (scientifique) à la phase 3 (commerciale) de la dynamique du processus d'innovation dans les IFS. Cette évolution de la situation de «concurrence-coopération» dans les IFS semble d'ailleurs statistiquement vérifiée par le déclin des accords à la fin des années 80 que l'on peut enregistrer à partir de la base de données CATI (Dodgson, 1992). Les implications en matière de stratégies technologiques de cette évolution de la situation de "concurrence-coopération», dans les IFS sont multiples. Il convient en particulier, aussi bien pour les grandes entreprises que pour les PME, de bien identifier les trois phases de la dynamique du processus d'innovation dans les IFS afin d'établir "à temps » le réseau de coopération qui leur permettra:

1. de s'assurer sur les futurs marchés et applications commerciales des positions stratégiques durables;

2. de disposer des actifs complémentaires;

3. de bénéficier suffisamment tôt des savoirs nécessaires;

4. d'identifier les voies technologiques prometteuses et celles qui sont sans avenir durable;

5. d'évaluer les premiers, le long des courbes d'apprentissage, quand les marchés et les applications deviennent effectifs ; cela d'autant plus qu'il s'agit de marchés fragmentés dont le taux de croissance est élevé.

Cette problématique du «temps optimal » en matière de partenariat est au cœur des stratégies technologiques de «création» et de «diffusion» de l'innovation dans les IFS. 


\section{Les stratégies technologiques de "création" et de diffusion des innovations dans les industries fondées sur la science}

La complémentarité stratégique existante entre grandes entreprises établies et petites firmes émergentes implique deux types de stratégies technologiques: les stratégies de «création» et de «diffusion» de l'innovation. Les stratégies technologiques de création de l'innovation concernent les développements scientifiques et technologiques qui ont pour objectif la réalisation des innovations de produits et de procédés. Les stratégies technologiques de diffusion de l'innovation concernent principalement l'utilisation des innovations de procédés issues des IFS. Stratégies de création et stratégies de diffusion impliquent des modes originaux de collaboration entre grandes entreprises et PME. Dans cette troisième partie, nous chercherons à expliciter ces modes de collaboration afin de définir le contenu particulier des stratégies technologiques, de création d'une part et de diffusion d'autre part, des innovations dans les industries fondées sur la science.

\subsection{Les stratégies technologiques de création de l'innovation}

Les acteurs industriels du processus de création des technologies dans les IFS peuvent être divisés en deux catégories (Hamilton, 1985):

- Les firmes établies, dans des marchés et des technologies existantes, qui sont (ou seront) confrontées aux «menaces» et aux «opportunités» induites par les nouvelles technologies.

- Les firmes émergentes, c'est-à-dire des petites firmes de haute technologie créées pour participer au développement de la nouvelle technologie considérée (biotechnologie, photonique, technologie de l'information, nouveaux matériaux).

Entre firmes établies et firmes émergentes, la complémentarité stratégique évolue au cours des trois phases du processus d'innovation selon une logique dégagée précédemment (diminution des «opportunités de coopération» et accroissement des «situations de concurrence»). Les manœuvres stratégiques des firmes établies et des firmes émergentes seront donc articulées d'après ces trois phases.

Les firmes établies, confrontées à une discontinuité technologique, ont le choix entre les manœuvres stratégiques suivantes (Hamilton, 1985; 1988).

- La «manœuvre de surveillance active» (window strategy) soit de façon interne (recherche exploratoire internalisée), soit par collaboration externe (subventions de recherche à des laboratoires publics 
ou privés, prises de participation dans les firmes émergentes). L'objectif de cette stratégie est d'accumuler de l'information sur la nouvelle technologie et ses implications commerciales à long terme, tout en limitant ses engagements à des ressources financières réversibles. Cette manœuvre est principalement adaptée à la phase 1 (scientifique), caractérisée par des collaborations dans des recherches «précompétitives » dont les objectifs sont très généraux.

- La «manœuvre de création d'options » suppose une participation plus active dans le développement de technologies et d'applications plus ciblées, soit de façon interne (R-D interne dans des directions précises), soit par collaboration externe (contrats de R-D en commun avec des firmes émergentes). Cette manœuvre stratégique est essentiellement caractéristique de la phase 2 (technologique).

- La «manœuvre de prise de positions » correspond à un objectif d'appropriation de certaines technologies et marchés. La grande entreprise peut prendre position de façon interne (création d'un atelier de production ou d'une petite unité autonome) ou de façon externe (prise de contrôle majoritaire ou totale d'une petite firme émergente). L'engagement de la firme en matière de ressources financières et humaines est plus élevé et difficilement réversible dans le cas de cette manœuvre stratégique qui convient à la phase 3 (commerciale).

Les firmes émergentes adoptent également des manoeuvres stratégiques (Hamilton, 1985 ; 1988) qui diffèrent avec le temps :

- La «manœuvre de création d'activités » caractérise essentiellement la première phase du processus d'innovation. La firme émergente doit se créer en tant qu'organisation autonome grâce à différentes ressources financières: capital de risque, contrats de recherches souvent publics, subventions.

- La «manœuvre d'extension des options » consiste à élargir son potentiel organisationnel par l'extension de ses activités de R-D rénumérées, des activités de conseil et même de formation, la réalisation de développements technologiques sous contrat, un recours accru au capital de risque, des R-D en commun et un positionnement exclusif sur des technologies choisies.

- La «manœuvre de prise de positions» est essentielle dans la phase 3 : il s'agit de s'approprier de façon exclusive, si possible, certaines niches de marché, tandis que pour certains produits des coentreprises ou des accords de partenariat peuvent être conclus avec des entre- 
prises établies afin d'assurer une commercialisation rapide à un niveau international.

En définitive, les stratégies technologiques de création de l'innovation dans les IFS impliquent des choix d'internalisation, de coopération et d'externalisation adaptés aux différentes phases du processus d'innovation dans ces industries.

Les petites firmes émergentes disposent d'atouts non négligeables dans ce jeu de «concurrence-coopération » à condition de bien «manager l'organisation du temps optimal» de leurs opérations financières, le choix de leurs partenaires, la négociation des clauses contractuelles, etc. Les exemples de réussites ou d'échecs dans le management de ces opérations financières et contractuelles abondent :

- Amgen (première entreprise de biotechnologie en 1993) a pu dépasser le leader Genentech, en partie, parce qu'elle a su profiter de l'engouement pour le capital de risque, pour les biotechnologies, au début des années 80 (Amgen a accumulé 19 millions de dollars auprès de fonds de capital de risque et de la firme pharmaceutique Abbott en 1981) puis collecter des fonds considérables sur le marché financier (150 millions de dollars entre 1983 et 1989) et cela avant d'enregistrer les premières recettes commerciales induites par le succès de son premier produit sur le marché (l'Epogen en 1989) (Constanty, 1993).

- Genentech en 1990 a négocié le respect de son autonomie de gestion et son indépendance en matière stratégique auprès d'Hoffmann la Roche, bien que cette grande entreprise ait acquis une participation majoritaire (60\% du capital) en injectant plus de 2 milliards de dollars tout en acceptant de ne prendre que deux sièges sur les treize existants du conseil d'administration de cette petite firme émergente, laquelle disposait essentiellement de produits en phase clinique (stade le plus coûteux du développement). Par la suite, Genentech a pu faire preuve de son indépendance en passant librement d'autres accords avec d'autres laboratoires comme Elli Lily, Kabi Pharmacia, Bayer... (Filloux, 1990; Ducruet, 1993).

- Dans le domaine de la photonique, la petite firme émergente allemande, Rofin-Sinar, doit son succès international à son intégration dans le groupe Siemens, tandis que les multiples accords établis en Europe par la firme américaine Coherent au début de 1980, et dont Rofin-Sinar commercialisait auparavant les sources, n'ont pas permis à Coherent d'empêcher le leadership de Rofin-Sinar de s'affirmer à son détriment à partir de 1985 (Larue de Tournemine, 1988). On peut 
avancer que Coherent aurait, sans doute, mieux protégé ses intérêts stratégiques si elle avait pris une participation dans le capital de Rofin-Sinar au début des années 80 .

\subsection{Les stratégies technologiques de diffusion de l'innovation}

Les stratégies technologiques de diffusion de l'innovation impliquent deux catégories d'utilisateurs possibles des innovations de procédé issues des industries fondées sur la science.

1. Les grandes entreprises établies sont susceptibles d'utiliser ces innovations de procédé pour reconfigurer leur processus de production. Mais bien souvent, ces grandes entreprises ne disposent ni de l'expertise nécessaire pour évaluer le bien-fondé de l'investissement dans le nouveau procédé, ni de la main-d'œuvre spécialisée pour assurer la mise en œuvre de ces innovations de procédé.

2. Les petites entreprises sous-traitantes (la sous-traitance «spécialisée ») qui ont fondé leur création et leur croissance sur une expertise affirmée dans l'utilisation de ces innovations de procédé.

Pour illustrer cette problématique de la complémentarité stratégique entre petites entreprises de sous-traitance spécialisée et grandes entreprises établies, prenons le cas de l'usinage laser. La décision d'investissement dans un système d'usinage laser est souvent difficile, car elle doit tenir compte de multiples paramètres dont le poids varie selon l'application et le contexte industriel considéré (Larue de Tournemine et al., 1993). Le processus décisionnel en matière d'investissement laser peut varier du simple (seule solution possible, faisabilité technico-économique établie) au très complexe (validation technico-économique difficile, incertitudes diverses). Pour le cas d'applications dont la validation technico-économique comporte un degré de risque élevé, les grandes entreprises peuvent se montrer réticentes à investir pour éviter de perturber leur processus de production. Dans ces cas, elles peuvent recourir aux petites entreprises spécialisées en sous-traitance technique dans le domaine de l'usinage laser. La complémentarité stratégique entre grandes et petites firmes dans la diffusion de l'innovation technologique et dans les industries fondées sur la science peut ainsi être illustrée par le rôle joué par les petites firmes sous-traitantes en usinage laser:

- Faisabilité technique et réalisation de prototype. Les entreprises sous-traitantes peuvent réaliser, pour le compte des grandes entreprises, les études de faisabilité préalables à l'achat d'un laser. Dans bien des cas, le recours à l'usinage laser permet de «re-concevoir» 
le produit usiné et les petites entreprises sous-traitantes réalisent les travaux d'analyse de la valeur du produit amélioré.

- Évaluation technico-économique. Les entreprises sous-traitantes spécialisées en usinage laser maîtrisent l'information concernant les performances techniques (vitesse, flexibilité...) et les paramètres économiques (coûts fixes et variables selon les applications et le type de machine laser) de l'investissement laser qui ne sont pas toujours du domaine public. Le recours à la sous-traitance spécialisée est ainsi, pour les grandes entreprises, un facteur de réduction des incertitudes technico-économiques concernant les applications d'usinage laser dans leur domaine d'activité.

- Réalisation de préséries. L'évaluation technico-économique peut nécessiter de simuler les conditions de production en série limitée selon un rythme de renouvellement adapté aux conditions de production de la sous-traitance (le rythme moyen du changement des séries en sous-traitance laser est inférieur à une heure).

Le recours à la sous-traitance technique en usinage laser est donc un facteur de réduction des incertitudes techniques et économiques pour les grandes entreprises. Inversement, diverses entreprises françaises de soustraitance en usinage laser ont pu effectuer les investissements nécessaires (la plupart des machines laser représente un coût supérieur à un million de francs) dans le cadre de plans de capitalisation auxquels ont participé des grandes entreprises intéressées par les prestations offertes par la soustraitance technique en usinage laser.

C'est, par exemple, le cas de la société ARES en France, fondée en 1986, pour des applications en usinage laser avec l'appui de grandes entreprises de l'aéronautique. L'appui de ces entreprises de l'aéronautique a été double (Larue de Tournemine et al., 1993):

- participation à la dotation en capital de la société ARES ;

- ouverture de débouchés assurés pour la société ARES, dans le cadre du rapatriement de la sous-traitance réalisée auparavant à l'étranger (États-Unis et Pays-Bas) pour un usinage de pointe (microperçage pour le refroidissement des aubes de réacteur pour l'aéronautique). 


\section{Conclusion}

Les diverses analyses effectuées dans cet article auront atteint leur but, si elles ont convaincu le lecteur d'envisager la problématique des stratégies technologiques dans les industries fondées sur la science, sous l'angle de l'évolution du processus d'innovation caractéristique de ces industries. Un aspect important de ce processus se trouve, par exemple, dans l'évolution de la «situation de concurrence-coopération» induite par le stade de développement de la technologie concernée. Les choix d'internalisation, les modes de coopération et les choix d'externalisation appropriés sont variables : ils dépendent autant pour les grandes entreprises que pour les petites de la phase (scientifique, technologique, commercial) de maturation des connaissances scientifiques, du degré de développement des technologies et d'identification des marchés et des applications. Les modes de collaboration appropriés sont «ouverts » et orientés par des objectifs de découverte et d'apprentissage en commun, au début du processus (phase 1 et 2). Quand le processus d'innovation est plus avancé (phase 3), les modes de collaboration appropriés sont plus exclusifs et orientés par l'objectif d'appropriation des bénéfices de l'innovation. Si tel n'est pas le cas, les bénéfices de l'innovation peuvent revenir à des firmes qui n'ont pas contribué aux avancées scientifiques et technologiques initiales, mais qui ont su créer les marchés et développer les applications au cours de la phase 3 (commerciale). Cette éventualité est observable dans le domaine de la photonique où le leadership incontesté des firmes américaines dans les phases 1 et 2 a finalement bénéficié en bonne partie aux firmes allemandes et japonaises dans la phase 3 (Larue de Tournemine, 1988). L'étude de ces phénomènes de renversement du leadership industriel et l'analyse des modalités de collaboration appropriées à chacune des phases du processus d'innovation dans les industries fondées sur la science sont encore des domaines de recherche à approfondir en matière de management et de stratégies technologiques.

Dans un autre domaine du management des technologies, celui du diagnostic concernant la position technologique respective des firmes dans un domaine d'activité considéré, il convient sans doute également de poursuivre les recherches et de proposer d'autres outils de diagnostic. Ceux-ci, en particulier, dans les industries fondées sur la science, pourraient compléter les outils qui existent pour apprécier la maîtrise interne d'une liste de ressources technologiques données (Morin, 1985). Ces nouveaux outils devraient être fondés sur une méthode d'analyse des coopérations établies (modalités, types et clauses particulières des accords) permettant d'apprécier la situation de chaque firme, dans le cadre du réseau de coopération existant, au moment du diagnostic dans le domaine technologique considéré. 


\section{Bibliographie}

ABERNATHY, W.J. et J.M. UTTERBACK (1978), «Pattern of industrial innovation », Technology Review, juin-juillet.

ACS, Z.J. et D. AUDRETSCH (1988), «Innovation in large and small firms: an analysis », American Economic Review, vol. 78, ${ }^{\circ} 4$, septembre.

AKRICH, M., M. CALlon et B. LAtour (1988), «À quoi tient le succès des innovations : l'art de l'intéressement », Annales des Mines, septembre.

BENASSI, M. (1993), «Organizational perspectives of strategic alliances : external growth in the computer industry » dans The Embedded Firm, Londres et New York, G. Grabher, Routledge (éd.).

BREndlé, CoHendet, GAFFARD et LARUE DE TOURnemine (1983), Les enjeux industriels de la photonique, Paris, La Documentation française, Ministère de l'Industrie et de la Recherche.

Callon, M. et B. Latour (1985), «Les paradoxes de la modernisation. Prospective et Succès », Publications du Centre de Sociologie de l'Innovation de l'École des Mines de Paris.

Constanty, H. (1993), «Amgen le petit prodige des bios», L'expansion, mai-juin.

CREPON (1991), «Taille des firmes et activité innovatrice», dans Innovation et Compétitivité sous la coordination de D. Guellec, INSEE Méthodes, n³7-38.

Dodgson, M. (1992), «The strategic management of R-D collaboration», Technology Analysis \& Strategic Management, vol. 4, $\mathrm{n}^{\circ} 3$.

Ducos, C. et P.B. Joly (1988), Les biotechnologies, Paris, Éditions La Découverte, Collection Repère.

Ducruet, C. (1993), «Pharmacie, biotechnologies : le temps des alliances », La Tribune Desfossés, 29 novembre.

FILlOUX (1990), «Genentech met ses labos sous un parapluie suisse », Libération, 12 février.

GAILlaRD, M.L. (1990), «Analyse de l'importance des technologies duales : les lasers et l'optoélectronique active », Rapport du CREST, Paris.

GEOFFRON (1991), «Une analyse du processus de structuration des industries du capital-risque», Revue Internationale PME, vol. $4, \mathrm{n}^{\circ} 3$.

HAGEDOORN, J. (1993), «Strategic technology alliances and modes of cooperation in high-technology industries », dans The Embedded Firm, Londres et New York, G. Grabher, Routledge (éd.).

HAMILTON, W.F. (1985), «Corporate strategies for managing emerging technology », Technology in Society, vol. 7. 
HAmilton, W.F. (1988), «The dynamics of technology and strategy », Communication au colloque EURO IX - TIMS XXII, Université Paris-Dauphine.

HLADIK, K.J. (1985), International Joint Ventures, New York, Lexington Books.

Horwitch, M. et C.K. Pralahad (1976), «Managing technological innovation: three ideal mods », Sloan Management Review, hiver, vol. 17.

KAMIEN, M. et N.L. SCHWARZ (1975), « Market structure and innovation : a survey », Journal of Economic Literature, $\mathrm{n}^{\circ} 1$.

LARUE DE TOURnEMINe, R. (1984) avec la collaboration de J. Bitoune, Les applications potentielles du laser d'usinage dans la CEE, Paris, publié par l'AREPIT.

LARUE DE TOURNEMINE, R. (1988), Les lasers d'usinage : applications, perspectives et stratégies technologiques, Paris, publié par l'AREPIT.

LARUE DE TOURNEMINE, R. (1991), Stratégies technologiques et processus d'innovation, Paris, Les éditions d'Organisation, Collection Campus Entreprise Université.

LARUE DE Tournemine, R. (1993) avec la collaboration de G. Lambert et P. Nanopoulos, «Économie et gestion dans l'insertion de l'outil laser», dans Étude de l'Insertion de l'outil laser dans les entreprises, Paris, Publication du Club Laser de Puissance.

LE BAS, C. (1991), «Économie du changement technique », L'Interdisciplinaire, Collection Technologies.

LITTLER, D. et D. WILSON (1990), «The evolution and strategic management of new technology - based sectors: the case of computerized business systems ", Technology Analysis \& Strategic Management, vol. 2, $\mathrm{n}^{\circ} 2$.

MAIDIQUE, M.A. (1980), «Entrepreneurs, champions and technological innovation », Sloan Management Review, vol. 20, $\mathrm{n}^{\circ} 2$.

MAIDIQUE, M.A. et R.H. HAYES (1984), «The art of high technology management », Sloan Management Review, hiver, vol. $25, \mathrm{n}^{\circ} 2$.

MoORE, W.L. et M.L. TUSHMAN (1982), « Managing innovation over the product life cycle», Reading in the Management of Innovation, Pitman Books Ltd.

MORIN, J. (1985), L'excellence technologique, Éditions J. Picollec.

PAVITT, K. (1984), « Sectoral patterns of technical change : towards a taxonomy and a theory », Research Policy, vol. 13.

PORTER, M.E. (1982), Choix stratégique et concurrence, Paris, Economica.

NGS, Pearson, A.W. et D.F. BalL (1992), «Strategies of biotechnology companies », Technology Analysis \& Strategic Management, vol. 4, ${ }^{\circ} 4$.

Rothwell, R. (1985), «Les petites et moyennes entreprises, moteur de l'innovation », La Recherche Économie, Supplément au n 183. 
TEECE, D.J. (1986), «Profiting from technological innovation: implications for integration, collaboration, licencing and public policy », Research Policy, vol. 15.

Zuscovitch, E. (1984), Une approche mésoéconomique du progrès technique: diffusion de l'innovation et apprentissage industriel, Thèse d'État, Strasbourg. 